\title{
Measuring Induction Times and Crystal Nucleation Rates
}

\author{
Clément Brandel ${ }^{\mathrm{a}}$, Joop H. ter Horst ${ }^{\mathrm{b}}$ * \\ DOI: 10.1039/b000000x [DO NOT ALTER/DELETE THIS TEXT]
}

${ }_{5}$ A large variation is observed in induction times measured under equal conditions in $1 \mathrm{ml}$ solutions. Ruling out experimental errors, this variation originates from the nucleation process. The induction time distribution is explained by the stochastic nature of nucleation if the number of nuclei formed is approaching 1 per vial. Accurate heterogeneous crystal 10 nucleation rates were determined from the induction time distributions on a $1 \mathrm{ml}$ scale for racemic Diprophylline in two solvents. The difference in nucleation behaviour in the two solvents originates from the energy barrier for nucleation, which is much higher in the solvent in which induction times are much longer. In addition the pre-exponential factor for the crystal 15 nucleation rate in both solvents is rather low compared to predictions using Classical Nucleation Theory. Unfortunately, concentration and surface characteristics of the effective heterogeneous particles are not known which clouds a further molecular interpretation.

\section{Introduction}

${ }_{20}$ Crystal nucleation rate predictions still are unreliable, differing orders of magnitude with the experimental ones [1,2]. At the same time, crystal nucleation rates are difficult to measure. This is due to the strongly non-linear behaviour making it necessary to accurately control supersaturation. Further, the nucleus, generally of a size of 1-1000 molecules, can as yet not be observed experimentally due to 25 analytical limitations and the difficulty in the localization of the nucleus. Another complication is the occurrence of growth, agglomeration and secondary nucleation during the primary crystal nucleation measurement, which can distort the experimentally obtained nucleation rates.

Accurately known crystal nucleation rates as a function of supersaturation under 30 well-defined conditions would enable a molecular level interpretation of the nucleation process [3]. Thorough nucleation rate measurements using the double pulse technique [4] for instance lead to the experimental identification of the twostep crystal nucleation mechanism [5]. Such molecular level interpretations will lead to the development of new crystal nucleation theories with improved predictive ${ }_{35}$ power.

Recently, a new measurement method was proposed that determines the crystal nucleation rate in stirred solutions using the variations in induction time measurements under equal conditions in small volumes [6]. The aim of this paper is to discuss the limitations and advantages of the new method. First, induction time 40 measurements under equal conditions are discussed after which it is shown that a conventional analysis does not arrive at reasonable nucleation rates. It is then shown that in these measurements a single nucleus mechanism is occurring for which a model is derived. The model can be used to determine nucleation rates from the

[journal], [year], [vol], 00-00 | 1 
obtained induction time distributions. Nucleation rates of the compound Diprophylline are analysed and discussed in the light of the Classical Nucleation Theory.

\section{Experimental}

5 Racemic Diprophylline (Sigma-Aldrich, $\geq 99 \%$ ), Isonicotinamide (Sigma-Aldrich, $\geq 99 \%$ ), 4-hydroxyacetophenone (Fluka Analytical, $\geq 98 \%$ ), ethanol (Sigma-Aldrich, $\geq 99.8 \%$ ), isopropylalcohol, dimethylformamide and ethyl acetate (J.T. Baker, $\geq 99.5 \%$ ) were used as received. Saturation temperatures and induction times were measured using a $1.5 \mathrm{~mL}$ multiple reactor system (Crystal16, Avantium Amsterdam).

10 It can hold 16 standard HPLC glass vials $(1.8 \mathrm{~mL})$ and measures the transmission of light through a sample in the vials. A temperature recalibration of the Crystall 6 was performed to make sure the actual temperature within the vials at the constant temperature of the induction time measurement coincided with the set temperature of the device. This means that the difference in the constant temperature between 15 vials was brought back to its minimum. Erlenmeyer flask, volumetric flask, pipettes, funnels and spatula were cleaned with $95 \%$ ethanol and dried prior to use to minimize the introduction of particulates and dissolved impurities.

To reduce concentration differences between sample vials a $25 \mathrm{ml}$ solution was prepared by dissolving the right amount of solid material in the solvent at elevated 20 temperature. A heated bottle-top dispenser was used to dispense $1 \mathrm{~mL}$ of clear solution into each vial. A small magnetic stirrer was introduced in each vial and the vial caps were strongly fixed to avoid evaporation of the solvent. They were then placed in the Crystall 6 setup.

A heat-hold-cool cycle was repeated at least 4 times with a controlled stirring ${ }_{25}$ speed of $700 \mathrm{rpm}$. Firstly, the sample was heated with a rate of $0.3^{\circ} \mathrm{C} / \mathrm{min}$ to $60^{\circ} \mathrm{C}$, which was well above the saturation temperature. Then, this temperature was maintained for 60 minutes to make sure that crystals were dissolved to obtain a clear solution. Then, the clear solution was cooled down to a constant temperature with a fast cooling rate of $5^{\circ} \mathrm{C} / \mathrm{min}$. The moment the set temperature was reached was taken 30 as time zero, after which the constant temperature was maintained for at least 5 hours. The induction time was taken as the difference between the time at which transmission of light started to decrease and time zero. The start and end time could be determined with an accuracy of 1 second. After 5 hours a subsequent heat-holdcool cycle was started. In this way a total of at least 64 induction time measurements 35 were obtained per supersaturation ratio value.

The vials were weighted before and after the experiments in order to check that evaporation of the solvent had not taken place. In the case the difference of weight was significant $(\geq 0.05 \%)$ the corresponding induction times were not considered in the analysis.

40 Care was taken to measure model systems that were not prone to crowning behaviour where a circle of crystals would form just above the liquid level. This was also checked during the measurements.

\section{Crystal Nucleation Rate Measurement Method}

\subsection{Induction times}

${ }_{45}$ The induction time $t_{\mathrm{i}}$ is defined as the time progress between the instance of constant

2 | [journal], [year], [vol], 00-00

This journal is @ The Royal Society of Chemistry [year] 
supersaturation creation and the instance of detection of crystals [7]. Figure 1 shows a typical induction time measurement on a $1 \mathrm{ml}$ scale in a stirred supersaturated solution. The instance of reaching constant supersaturation was chosen to be the moment that the set temperature reached the desired constant temperature. The 5 actual temperature in the vial will probably show a slight delay. Therefore, series of measurements in which induction times of close to zero are measured should be carefully treated.

The response of the transmission of light to the presence of crystals is quite sharp. However, the transmission of light detects does not detect the nucleus. It detects the 10 moment that the suspension contains a certain volume fraction of crystals. Therefore, the moment that the transmission of light decreases is delayed in respect to the moment that the nucleus appears.

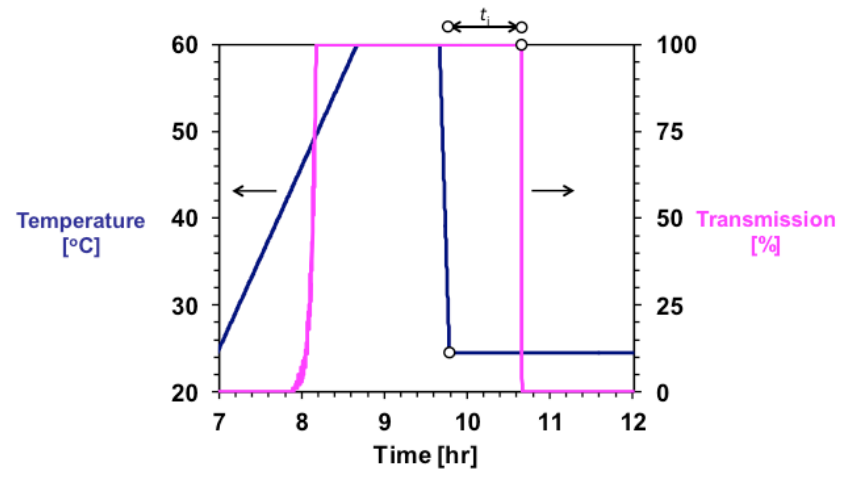

Figure 1: An induction time $t_{\mathrm{i}}$ measurement using temperature to control supersaturation and light to detect crystals. The start of the $t_{\mathrm{i}}$ measurement, indicated by the circle on the temperature line, is the time of reaching the set temperature of $25^{\circ} \mathrm{C}$. The end of the measurement, indicated by the circle on the light transmission line, is the time at which crystals are detected by a decrease in the transmission of light through the sample. The measured induction time was roughly 2900 seconds.

Figure 2 shows the induction times measured for two vials in 8 consecutive experiments under equal conditions. While the induction time can be measured 15 under well-defined and well-controlled conditions, a large variation in the induction time values can be seen. The series of measurements show induction times respectively ranging from 156 to $3044 \mathrm{~s}$ and from 390 to $1673 \mathrm{~s}$.

The induction time variations within a measurement series in a single vial, therefore, show large variations. Weighing the vials before and after the series of ${ }_{20}$ experiments indicated that no solvent evaporation took place. Solvent evaporation would lead to an increase in supersaturation during the series of measurements and thus to a decreasing induction time throughout the series of measurements. Also the measured clear point of the formed suspensions did not show changes and thus did not indicate a change in supersaturation. Also no indications of decomposition of ${ }_{25}$ solvent or model compound were obtained.

Both series of measurements seem to vary similarly. These variations are not the result of concentration differences between the vials or within the series. Buffer solutions were used for the vials making sure that the concentration difference between the vials was as small as possible. It seems that the variations are a result of 30 the crystallization in the vials.

[journal], [year], [vol], 00-00|3

This journal is $\odot$ The Royal Society of Chemistry [year] 


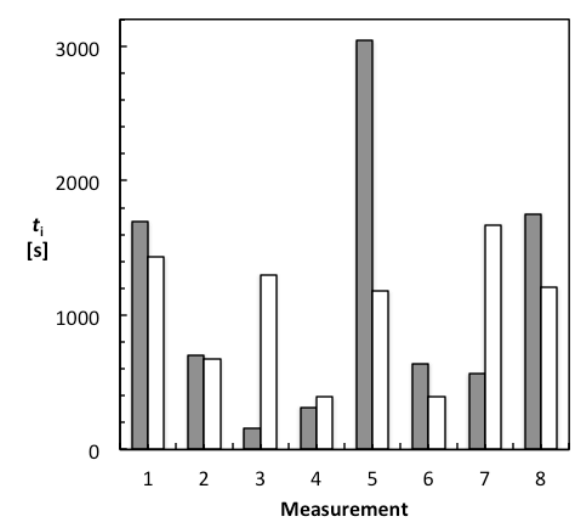

Figure 2: Two series of 8 subsequently measured induction times under equal conditions in a single vial. The system is 4-hydroxy acetophenone in ethyl acetate at a supersaturation of $S=1.3$.

\subsection{The Conventional Model for Induction Time Analysis}

One way of analysing induction times is to assume detection upon filling of a certain fraction of the volume by the crystalline phase. If it is assumed that the suspension is formed by only primary nucleation and growth, the induction time can be written as 5 [7]:

$$
t_{i}=\left(\frac{3 \alpha}{\pi G^{3} J}\right)^{1 / 4}
$$

Where it was assumed that spherical particles are formed which grow with a linear growth rate $G$. The equation further contains the detectable crystal volume fraction $\alpha$ and the crystal nucleation rate $J$. The nucleation rate $J$ can be determined from the 10 induction time $t_{\mathrm{i}}$ by assuming a value for the growth rate $G$ and volume fraction $\alpha$.

$$
J=\frac{3 \alpha}{\pi G^{3} t_{i}^{4}}
$$

By assuming a value of $G=10^{-7} \mathrm{~m} / \mathrm{s}$ and of $\alpha=0.1$ we arrive at crystal nucleation rates varying from 1 to $161.000 \mathrm{ml}^{-1} \mathrm{~s}^{-1}\left(10^{6}\right.$ to $\left.161.10^{9} \mathrm{~m}^{-3} \mathrm{~s}^{-1}\right)$ for the induction times in Figure 2. This is a variation over 5 orders of magnitude. These variations in 15 nucleation rate cannot be explained by variations in conditions such as temperature and concentration since these were very small. The variations therefore would have to be the result of the crystallisation process itself. However, having these kinds of induction time variations for nucleation rates of more than 1 per vial per second seems not possible because of the large number of crystals that would be present in 20 the vials, diminishing stochastic behaviour.

\subsection{The Single Nucleus Mechanism}

Visualization of the stirred solution in $3 \mathrm{ml}$ volumes showed the presence of a large single crystal before a crystal suspension was detected by the decrease in the transmission of light through the sample [8]. Figure 3 shows a clear solution, the ${ }_{25}$ single parent crystal and the suspension after detection through a decrease in the transmission of light. The observation of the single crystal relied on the probability that on the small number of occasions that when it passed the camera it also would be captured by the camera ( 1 picture per second). Probably therefore, not all

4 | [journal], [year], [vol], 00-00

This journal is ( ) The Royal Society of Chemistry [year] 
visualization experiments resulted in the observation of a single crystal. However, this single nucleus mechanism was observed on a $3 \mathrm{ml}$ scale in a large number of model systems, among which isonicotinamide in ethanol, butanol, nitrobenzene, nitromethane, paracetamol and succinic acid in water as well as 4-hydroxy 5 acetophenone in ethyl acetate. Increasing the solution volume at constant supersaturation decreases the induction time variation because more nuclei form in the solution. Similarly, variations in metastable zone widths reduced in larger volumes [9]. While for paracetamol in water there were no variations anymore on a 1 liter scale, there was still significant variation for isonicotinamide in ethanol on 10 the same scale.

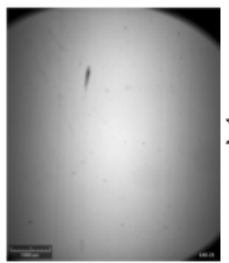

Clear solution

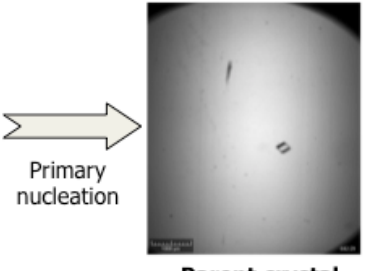

Parent crystal

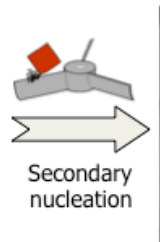

Secondary nation

Growth

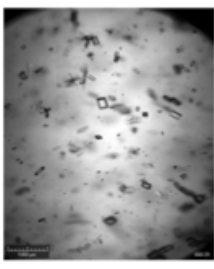

Suspension

Figure 3: The single nucleus mechanism where a single parent crystal nucleates in a clear solution. After growth of this parent crystal secondary nucleation occurs and a suspension is forming.

If the single crystal is large enough it can cause secondary nucleation either through crystal-stirrer collisions (attrition) or some surface breeding mechanism. Then, only a single crystal is responsible for the generation of the entire final crystal population in the suspension. Under concomitant polymorphism conditions of 15 isonicotinamide in solvent mixtures it was tested whether cooling crystallisation in 3 $\mathrm{ml}$ volumes resulted in pure polymorphic products or in polymorphic mixtures. A series of 10 stirred cooling crystallisation experiments lead to 7 of them crystallizing as pure form I and 3 as pure form II while no mixtures were observed [10]. Other systems showed similar behaviour.

20 These experiments indicate that a single nucleus mechanism is occurring. After supersaturation generation a single crystal nucleates and grows until it is large enough for secondary nucleation to occur. Then quickly a full suspension of crystals, all originating from the same single crystal is formed. The crystallisation process in such small volumes then is split up into different periods in which the subsequent ${ }_{25}$ processes of crystallisation occur. First, a single crystal nucleation event occurs. Then, growth occurs up to a macroscopic size. Finally, secondary nucleation occurs due to the presence of this large single crystal in the stirred solution. Since the subprocesses of crystallisation are effectively separated, a simple model can be made to capture this process as well as the variations in it.

\section{3.4. A Model for the Single Nucleus Mechanism}

The variation of induction times can be explained if the formation of the suspension is the result of a single nucleation event. This event depends on the probability of the formation of a nucleus in a certain timeframe. The lower the nucleation rate, the lower the probability would be to form a nucleus in this timeframe. At constant 35 supersaturation this can be captured through the Poisson distribution, which describes the probability $P_{\mathrm{m}}$ of the formation of $m$ nuclei in a certain timeframe $t$.

[journal], [year], [vol], 00-00|5 


$$
P_{m}=\frac{N^{m}}{m !} \exp (-N)
$$

Where $N(t)=J V t_{J}$ is the average number of nuclei formed in the timeframe $t_{J}$ with the rate $J$ and within a volume $V$. The probability $P^{*}(t)$ that one or more nuclei are formed is then:

${ }_{5} \quad P^{*}=1-P_{0}=1-\exp \left(-J V t_{J}\right)$

We can only detect a suspension of crystals occupying a fraction of the volume which gives a time delay $t_{\mathrm{g}}$, the difference between the time $t_{\mathrm{J}}$ and the time $t$ of detection of crystals. If this growth time is equal for all detections, the probability $P(t)$ that crystals are detected is given by:

$10 \quad P(t)=1-P_{0}=1-\exp \left(-J V\left(t-t_{g}\right)\right)$

The above equation is a description of the probability $P(t)$ that crystals are detected in a supersaturated solution sample as a function of time. It depends on the nucleation rate $J$, the sample volume $V$ and the growth time $t_{\mathrm{g}}$. This equation is valid starting at time $t_{\mathrm{g}}$. Below time $t_{\mathrm{g}}$ the probability $P(t)$ would be zero. For very large 15 times $t$ the probability would become 1 .

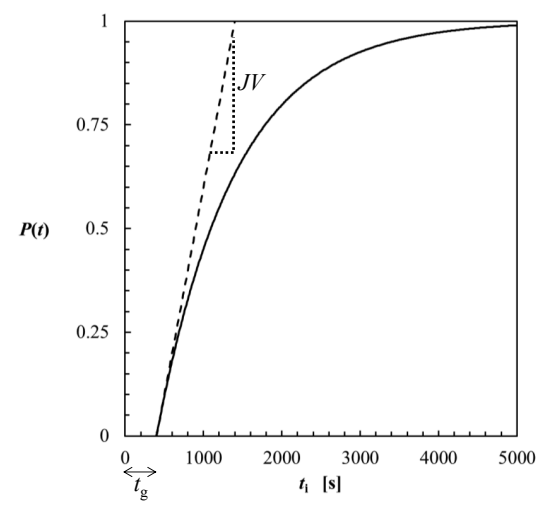

Figure 4: The probability from eq. (5) as a function of time. The relation between equation and graph is indicated by $t_{\mathrm{g}}$ and $J V$. The graph was constructed with a growth time $t_{\mathrm{g}}=400 \mathrm{~s}$ and product of volume $V$ and nucleation rate $J$ of $J V=0.001 \mathrm{~s}^{-1}$.

\subsection{Nucleation Rate Determination}

The probability $P(t)$ is also experimentally accessible. It can be determined by measuring a large number of induction times under absolutely equal conditions as we did in the case of HAP in ethyl acetate shown in Figure 2. The experimental 20 probability $P(t)$ can then determined from:

$$
P(t)=\frac{M^{+}(t)}{M}
$$

Where $M$ is the total number of induction time measurements and $M^{+}(t)$ is the number of measurements in which crystals are already detected at time $t$. Figure 5 shows a typical induction time distribution using a series of 120 induction times ${ }_{25}$ divided over 8 vials. The smallest induction time measured was $156 \mathrm{~s}$ while the largest was $4100 \mathrm{~s}$. After an initial time period within which no crystals were detected for all samples, the probability $P(t)$ quickly increases and levels off towards a probability of 1 . This is exactly the curve shape that is captured in the model for

6 | [journal], [year], [vol], 00-00

This journal is @ The Royal Society of Chemistry [year] 
the single nucleus mechanism proposed earlier.

As an example the cumulative probability distribution function is fitted to the experimentally determined probability distribution using a nucleation rate $J=865 \pm 20$ $\mathrm{m}^{-3} \mathrm{~s}^{-1}$ and growth time $t_{\mathrm{g}}=263 \pm 20 \mathrm{~s}$. Model and data can be fitted quite well, only ${ }_{5}$ for the higher values of $P(t)$ a deviation between them is apparent. It thus seems that the method can be used to determine crystal nucleation rates.

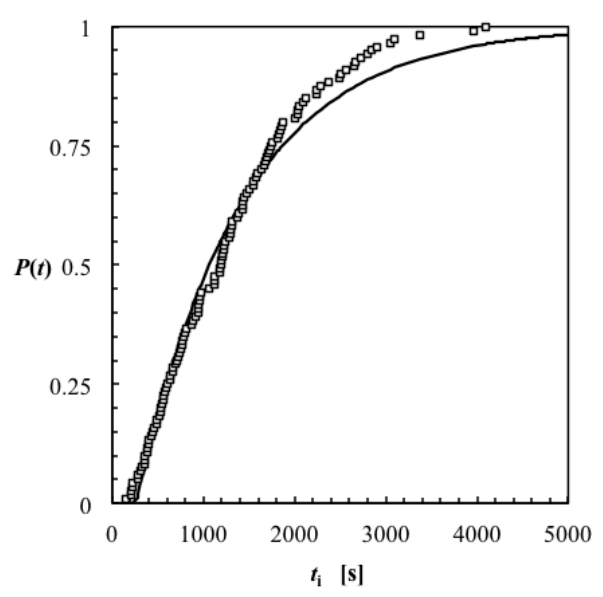

Figure 5: The induction time distribution of 120 induction times measured for HAP in ethanol at a supersaturation $S=1.3$ and temperature $T=25^{\circ} \mathrm{C}$. The distribution function (eq. (5)) was fitted using a nucleation rate $J=865 \mathrm{~m}^{-3} \mathrm{~s}^{-1}$ and a growth time $t_{\mathrm{g}}=263 \mathrm{~s}$.

\subsection{Heterogeneous Crystal Nucleation}

Usually it is assumed that crystal nucleation from solution occurs through heterogeneous nucleation: crystals form onto foreign particles such as dust particles 10 or on interfaces such as that between glass walls, stirrer surface or air and the solution. To investigate the kind of heterogeneous nucleation occurring during the crystal nucleation rate measurements using the model system isonicotinamide in ethanol 3 types of experiments were done. Next to the base experiments the solution was filtered or the vials were silanized. These 3 series of induction time 15 measurements are shown in Figure 6.

While the silanization of the glass surfaces of the vials does not seem to have an effect on the distribution, the solution filtration has a marked effect. Longer induction times are needed to reach the same induction time probability: apparently the nucleation rate decreased. It can be seen that this is the nucleation rate and not 20 the growth time because all probability curves intercept the time-axis at around the same point. It thus can be concluded that the crystal nucleation takes place on suspended heterogeneous particles in the solution that can be partially removed by solution filtration [11]. Unfortunately, concentration and surface functionality of these heterogeneous particles are not known. This is actually generally true for the ${ }_{25}$ heterogeneous particles in crystallisation processes.

[journal], [year], [vol], 00-00 7 


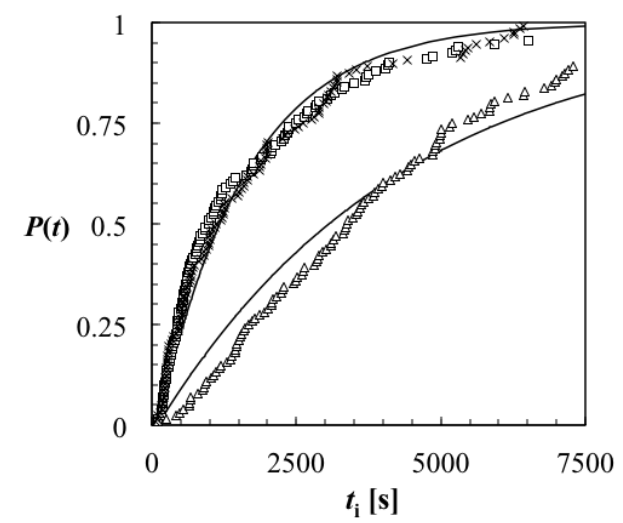

Figure 6: The induction time probability distribution of isonicotinamide in ethanol at a supersasturation of $S=1.44$. $\square$ : Untreated vial and solution; $\times$ : Using silanized vials; $\triangle$ : Using filtered solutions.

\section{Nucleation Rate Analysis \& Interpretation}

The theophylline derivate Diprophylline (DPL, Figure 7), used in the treatment of obstructive airway diseases such as bronchial asthma, is marketed as a racemic compound. Racemic DPL is a polymorphic compound [12] which can form as the 5 marketed stable form RI and a metastable form RII. Supersaturated DPL solutions have difficulty crystallizing showing in a persistence of the metastable supersaturated solution: It took at least 3 hours before crystals were observed in a stirred solution in Dimethylformamide (DMF) at $15^{\circ} \mathrm{C}$ at a supersaturation ratio $S=2.8$ [13]. While prone to large metastable zone widths, polar solvents like 10 Dimethylformamide (DMF) result in DPL form RI. In the less polar solvent DPL the metastable zone is much smaller while form RII crystallizes. At $20^{\circ} \mathrm{C}$ the solubility of RI in DMF is $7.0 \mathrm{w} \%$ while that of RII in IPA is $0.5 \mathrm{w} \%$. During the gravimetric solubility measurements no transformation of RII to RI occurred in IPA. Crystallization experiments showed that only form RII formed in IPA. In DMF only

15 form RI formed. To compare and analyse crystal nucleation rates for the different polymorphs we have measured induction time distributions of DPL from DMF and IPA.

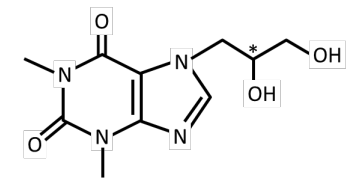

Figure 7: The molecular structure of Diprophylline.

\subsection{Induction time distribution measurements}

${ }_{20}$ Figure 8 shows a number of experimental induction time distributions for different supersaturation ratios of racemic DPL in IPA and DMF at $20^{\circ} \mathrm{C}$. It can be seen that

8 | [journal], [year], [vol], 00-00

This journal is $\odot$ The Royal Society of Chemistry [year] 
the distributions become broader at lower supersaturations indicating lower nucleation rates. The point where the probability starts to increase from zero is larger for smaller supersaturation ratios, indicating an increase in the growth time $t_{\mathrm{g}}$ with decreasing supersaturation ratios.

5 A remarkable difference between the 2 graphs in Figure 8 is the prevailing supersaturation ratio. While in IPA supersaturation ratios of $S=1.5$ to 3 are responsible for the measured distributions, in DMF they are $S=3$ and up. Looking at the measurements at $S=3$ in both graphs, for DPL in IPA and DMF induction times from respectively 5.9 to 173 and 43 to 424 minutes are measured. All curves possess 10 the typical distribution shape as in Figure 4. Fitting Eq. (5) to the experimental induction time distributions in Figure 8 gives the values in Table 1 for the nucleation rate $J$ and growth time $t_{\mathrm{g}}$ at different supersaturations. Apparently the nucleation of RII from IPA is much faster than the nucleation of RI (but also RII) from DMF. It is interesting to investigate whether this is due to the nucleation 15 kinetics or the energy barrier for nucleation.
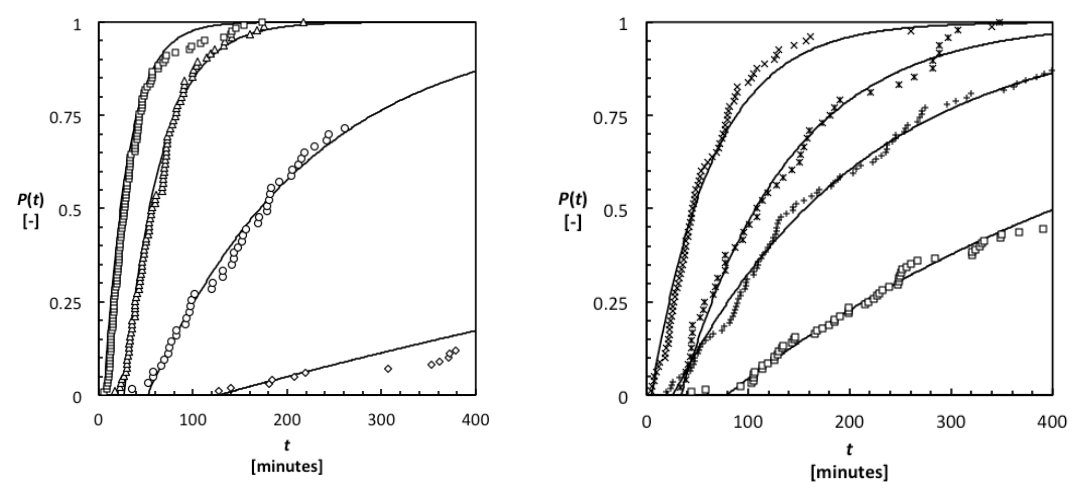

Figure 8: The experimental induction time probability distributions of Diprophylline (DPL) in IPA and DMF at different weight fraction-based supersaturation ratios $S$. Also the fits to eq. (5) are shown. Left: DPL in IPA: $\diamond-S=1.5, \bigcirc-S=1.7, \triangle-S=3.0, \square-S=3.0$; Right: DPL in DMF: $\square-$

$S=3.0,+-S=3.5, *-S=4.0, \times-S=4.2$. Note that time is given in minutes rather than seconds

Table 1: Crystal Nucleation rates $J$ and growth times $t_{\mathrm{g}}$ determined from the fit of eq. (5) to the experimental induction time probability distributions of DPL in IPA and DMF. The error in the fit is less than $10 \%$.

\begin{tabular}{crrrr}
\hline & \multicolumn{2}{c}{ DPL RII from IPA } & \multicolumn{2}{c}{ DPL RI from DMF } \\
\cline { 2 - 5 } $\begin{array}{c}S \\
{[-]}\end{array}$ & $\begin{array}{c}J \\
{\left[\mathrm{~m}^{-3} \mathrm{~s}^{-1}\right]}\end{array}$ & $\begin{array}{c}t_{\mathrm{g}} \\
{[\mathrm{min}]}\end{array}$ & $\begin{array}{c}J \\
{\left[\mathrm{~m}^{-3} \mathrm{~s}^{-1}\right]}\end{array}$ & $\begin{array}{c}t_{\mathrm{g}} \\
{[\mathrm{min}]}\end{array}$ \\
\hline 1.5 & 11.6 & 128 & & \\
1.7 & 97.0 & 52 & & \\
2.0 & 429 & 27 & & \\
3.0 & 668 & 5.9 & 35.7 & 79 \\
3.5 & & & 88.7 & 26 \\
4.0 & & & 157 & 34 \\
4.2 & & & 275 & 4.7 \\
\hline
\end{tabular}

\section{4.2. Nucleation Rate Factors}

The obtained crystal nucleation rates at different supersaturations can now be analysed in terms of the Classical Nucleation Theory. The crystal nucleation rate equation from Classical Nucleation Theory is given by [1,7]:

[journal], [year], [vol], 00-00|9 


$$
J=A S \exp \left(-\frac{B}{\ln ^{2} S}\right)
$$

Although also other dependencies of the pre-exponential term on the supersaturation ratio $S$ are known in literature [7,14]. The variables $A$ and $B$ are supersaturation ratio $S$ independent variables and can be referred to as the kinetic factor or pre${ }_{5}$ exponential factor $A$ and thermodynamic or exponential factor $B$. The equation can be rearranged:

$$
\ln \frac{J}{S}=\ln A-\frac{B}{\ln ^{2} S}
$$

This shows that the experimental nucleation rate data can be plotted as $\ln (J / S)$ versus $\ln ^{-2} S$ which would result in a straight line with intercept $\ln A$ and slope $-B$. This is ${ }_{10}$ done in Figure 9 for the nucleation rates reported in Table 1 of DPL in IPA and DMF. It can be seen that the points are to some extend on a straight line. The fitting procedure results in a value of $A=576 \mathrm{~m}^{-3} \mathrm{~s}^{-1}$ and $B=0.68$ for DPL from IPA and $A=499 \mathrm{~m}^{-3} \mathrm{~s}^{-1}$ and $B=4.57$ for DPL from DMF (Table 2). While the thermodynamic parameter $B$ is an order of magnitude larger for DPL in DMF compared to that in 15 IPA, the kinetic parameter is similar in size.

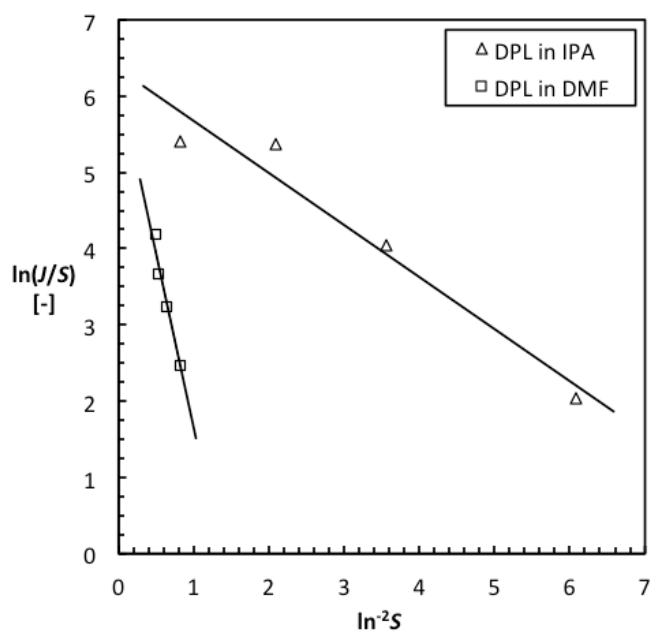

Figure 9: The experimental crystal nucleation rates of DPL from IPA and DMF (Table 1) plotted following Eq. (8) in order to determine the kinetic factor $A$ and the thermodynamic factor $B$.

10 | [journal], [year], [vol], 00-00 
Table 2: Values for the pre-exponential kinetic factor $A$ and the thermodynamic factor $B$ determined for a number of different systems using the same crystal nucleation rate measurement method.

\begin{tabular}{c|ccc}
\hline System & \multicolumn{3}{c}{ Reference } \\
& $\begin{array}{c}A \\
{\left[\mathrm{~m}^{-3} \mathrm{~s}^{-1}\right]}\end{array}$ & $B$ & This work \\
\hline DPL form RII in IPA & $576 \pm 235$ & $0.68 \pm 0.11$ & This \\
DPL form RI in DMF & $499 \pm 221$ & $4.57 \pm 0.70$ & This work \\
m-aminobenzoic acid in 50w\% water/ethanol & $870 \cdot 10^{3}$ & 3.6 & {$[6]$} \\
L-histidine in water & $36.3 \cdot 10^{3}$ & 1.1 & {$[6]$} \\
isonicotinamide in ethanol & $5.86 \cdot 10^{3}$ & 0.323 & {$[11]$} \\
p-aminobenzoic acid in acetonitrile & $36.3 \cdot 10^{3}$ & 0.016 & {$[14]$} \\
p-aminobenzoic acid in ethyl acetate & $20.5 \cdot 10^{3}$ & 0.053 & {$[14]$} \\
p-aminobenzoic acid in 2-propanol & $10.9 \cdot 10^{3}$ & 0.078 & {$[14]$} \\
benzoic acid in toluene & $61.8 \cdot 10^{3}$ & 0.46 & {$[14]$} \\
\hline
\end{tabular}

4.3. Interpretation of the Thermodynamic Factor $B$

The thermodynamic parameter $B$ is much higher for DPL nucleation from DMF than ${ }_{5}$ from IPA. This indicates that at the same supersaturation ratio $S$ the crystal nucleation work, which is the energy barrier for nucleation, is much larger for DPL nucleation from DMF. The nucleation work $W^{*}$ can be calculated using $W^{*} / k T=B / \ln ^{2} S$. For a supersaturation ratio of $S=3$ this gives values of $W^{*}=0.6$ and $3.8 k T$ for DPL nucleation from respectively IPA and DMF. These nucleation work 10 values seem on the low side, especially for DPL nucleation from IPA with its nucleation work below $1 k T$.

In section 3.4 it was established that heterogeneous rather than homogeneous nucleation takes place for isonicotinamide in ethanol. Most probably this is also the case for DPL. For heterogeneous nucleation the thermodynamic factor $B$ is related to 15 the creation and reduction of surface areas with different interfacial energies. It is a balance between the 3 interfacial energies that play a role: the interfacial energy between nucleus and heterogeneous particle, nucleus and solution, and solution and heterogeneous particle. This creates an energy barrier (nucleation work) lower than that for homogeneous nucleation in absence of a heterogeneous particle in which 20 only a single interfacial energy plays a role: that between the nucleus and the solution. Therefore, an effective interfacial energy $\gamma_{\text {ef }}$ can be introduced that captures the balance between all interfacial energies involved.

The relation between heterogeneous nucleation work $W^{*}$ and the thermodynamic parameter $B$ for heterogeneous nucleation is given by [1,7]:

$25 \quad \frac{W^{*}}{k T}=\frac{4 c^{3} v^{2} \gamma_{e f}}{27(k T)^{3} \ln ^{2} S}=\frac{B}{\ln ^{2} S}$

In which shape factor $c=(36 \pi)^{1 / 3}$ for a spherical particle, temperature $T=298 \mathrm{~K}$ and molecular volume $v=269 \times 10^{-30} \mathrm{~m}^{3}$ can be assumed for DPL to calculate the effective interfacial energy $\gamma_{\mathrm{ef}}=3.34$ and $6.29 \mathrm{~mJ} / \mathrm{m}^{2}$ from the thermodynamic parameters $B=0.323$ and 4.48 of DPL in respectively IPA and DMF. The effective 30 interfacial energy for DPL nucleation from DMF is much larger and therefore the nucleation work is higher and the nucleation proceeds much slower.

Crystal solubility is connected to the interfacial energy between crystal and solution [15]. The interfacial energy between DPL crystals and the solution can be calculated from the solubilities to be around $\gamma=29.9$ and $15.6 \mathrm{~mJ} / \mathrm{m}^{2}$ for DPL in ${ }_{35}$ respectively IPA and DMF. Since the solubility in IPA is much smaller than in DMF the interfacial energy is twice as large. Interestingly, while the interfacial energy $\gamma$ is much larger for DPL in the solvent IPA compared to the solvent DMF, the effective

[journal], [year], [vol], 00-00 | 11 
interfacial energy $\gamma_{\mathrm{ef}}$ is much smaller in IPA.

The relation between the interfacial energy $\gamma$ between crystal and solution and the effective interfacial energy $\gamma_{\mathrm{ef}}$ is given by the activity factor $\psi=\gamma_{\mathrm{ef}} / \gamma$. Effectively, the interfacial energy between crystal and solution is substantially decreased by the 5 presence of the heterogeneous particles. Table 3 shows the calculated values for DPL nucleation from IPA and DMF. The calculated value for the activity factor of $\psi=0.11$ and 0.40 seem characteristic values for heterogeneous crystal nucleation. They also seem to indicate that the heterogeneous particles in IPA are more effective in reducing the energy barrier comparted to those in DPL solutions in DMF. ${ }_{10}$ However, care should be taken with the method to determine interfacial energies from solubilities because it is based on a constant derived from supposedly homogeneous crystal nucleation rate measurements of ionic compounds [16].

Table 3: Interfacial energy $\gamma$ calculated from the solubility, effective interfacial energy $\gamma_{\text {ef }}$ from the nucleation rate measurements and activity factor $\psi=\gamma_{\mathrm{ef}} / \gamma$ for DPL in IPA and DMF.

\begin{tabular}{|c|c|c|c|}
\hline System & $\begin{array}{c}\gamma \\
{\left[\mathrm{J} / \mathrm{m}^{2}\right]}\end{array}$ & $\begin{array}{c}\gamma_{e f} \\
{\left[\mathrm{~J} / \mathrm{m}^{2}\right]}\end{array}$ & $\psi=\gamma_{e f} / \gamma$ \\
\hline $\begin{array}{l}\text { DPL form RII in IPA } \\
\text { DPL form RI in DMF }\end{array}$ & $\begin{array}{l}29.9 \\
15.6\end{array}$ & $\begin{array}{l}3.34 \\
6.29\end{array}$ & $\begin{array}{l}0.11 \\
0.40\end{array}$ \\
\hline
\end{tabular}

\section{4.4. Interpretation of the Kinetic Factor $A$}

The pre-exponential factor $A$ found for DPL is roughly equal in both solvents. The theoretical value of the kinetic factor $A$ is estimated to be $10^{15}-10^{25} \mathrm{~m}^{-3} \mathrm{~s}^{-1}[7,15]$. This is orders of magnitude larger than the experimental values for $A$ of DPL nucleation. Other compounds also show low pre-exponential factors ranging from $2010^{3}$ to $10^{6} \mathrm{~m}^{-3} \mathrm{~s}^{-1}$. The experimental kinetic factor $A$ is thus orders of magnitude smaller than theoretically is expected. In order to analyse this discrepancy the different parts that constitute the kinetic factor should be looked at. The preexponential term $A S$ from eq. (8) describes the statistical process that a building unit attaches to the nucleus [1]. It is the product of the concentration of nucleation sites ${ }_{25} C_{0}$, the attachment frequency $f^{*}$ and the Zeldovich factor $z$.

$$
A S=z f^{*} C_{0}
$$

The Zeldovich factor $z$ expresses that clusters larger than the nucleus size still have a probability to decay rather than to grow out to macroscopic sizes [1]. It further corrects for the use of an equilibrium nucleus concentration rather than the 30 actual nucleus concentration in the derivation of the crystal nucleation rate equation. It does not seem likely that the Zeldovich factor is the origin of the discrepancy between theoretical and experimental values.

The attachment frequency $f^{*}$ of building units to the nucleus determines how many nuclei of size $n^{*}$ chance to supernuclei of size $\left(n^{*}+1\right)$ per unit of time. During 35 this process, building units must transfer from being dissolved and solvated to being partially desolvated and adsorbed on the nucleus surface. For an interfacial transfer controlled attachment frequency, the rate that building units become adsorbed can be given by $[1,7]$ :

$$
f^{*}=\lambda A^{*} D \frac{X}{d}
$$

${ }_{40}$ Where $A^{*}$ is the nucleus surface area, $X$ is the concentration of building units in the solution and $d$ reflects a distance representing the jump of the building unit from dissolved to partially adsorbed. The parameter $D$ is some kind of diffusion

12 | [journal], [year], [vol], 00-00 
coefficient that describes the transfer of a building unit from the nucleus neighbourhood to being partially adsorbed. The sticking coefficient $\lambda$ reflects that there are building units in the nucleus neighbourhood not transferring to being adsorbed.

5 The attachment frequency thus depends on how the solute is present in the solution. The solute might have a solvent shell that has to be removed before it can be partially adsorbed onto the nucleus. There could also be different associates present in solution that differ in concentration $X$ and diffusion coefficient $D$. Isonicotinamide for instance shows widely different self-association behaviour in 10 different solvents resulting in varying polymorphic crystallisation outcomes [17].

If the discrepancy between theoretical and experimental $A$ comes from the attachment frequency $f^{*}$, the sticking coefficient $\lambda$, the concentration $X$ or the diffusion coefficient $D$ would be orders of magnitude smaller than theoretically predicted. Since this attachment process is difficult to access in experiments, the 15 study of it with molecular simulations would enable a more accurate and sound description, leading to increased understanding and predictability of crystal nucleation rates.

The concentration of nucleation sites $C_{0}$ is related to the concentration of heterogeneous particles. If only 1 nucleus forms on a heterogeneous particle, the ${ }_{20}$ concentration $C_{0}$ coincides with that of the heterogeneous particles. Apart from their concentration, their surface functionality is important since it influences the interfacial energies involved in the heterogeneous nucleation work, the effective interfacial energy, the activity factor and thus the thermodynamic factor $B$. From the analysis of the factor $B$ we found an activity factors of $\psi=0.11$ and 0.40 .

${ }_{25}$ Unfortunately, the concentration of particles onto which crystal nucleation occurs is not known. We can say that if the discrepancy between theoretical and experimental $A$ comes from the concentration of nucleation sites, the actual concentration of nucleation sites is order of magnitude smaller than assumed in the theoretical prediction. Anyway, the heterogeneous particles responsible for heterogeneous ${ }_{30}$ nucleation cannot be separately characterized. This makes it impossible to interpret the concentration of nucleation sites $C_{0}$ and with it, the kinetic factor $A$.

The way forward in crystal nucleation research lies in the interpretation of crystal nucleation rate data. However, a good interpretation cannot be done without knowing anything about the effective heterogeneous particles and the self35 association and solvation processes in the solution. A first attempt was given for isonicotinamide of which the role of self-association on template crystallisation was recently studied [18].

The nucleation rate equation from Classical Nucleation Theory is backed by a thorough and detailed physical description [7]. Apparently, this description does not 40 significantly accurate capture the true molecular aspects of the process. We would first have to establish the deficits in this description by Classical Nucleation Theory before developing new predictive theories.

\section{Conclusions}

The variation in induction times of experiments under equal conditions is resulting ${ }_{45}$ from crystal nucleation behaviour. There seems to be a single nucleus mechanism in operation where a parent crystal is formed which, through secondary nucleation, is responsible for the formation of the suspension. The proposed crystal nucleation

[journal], [year], [vol], 00-00|13 
measurement method based on induction time probability distributions allows the determination of the nucleation rate as a function of supersaturation. It is established that heterogeneous nucleation on particles in the solution is occurring.

The crystal nucleation rates of DPL from IPA and DMF were measured as a ${ }_{5}$ function of the supersaturation. The thermodynamic factor $B$ in the nucleation rate equation was determined to be much higher for DPL in DMF than for DPL in IPA. Therefore, the energy barrier for nucleation is much higher in DMF than in IPA. The analysis of the kinetic factor $A$ of the nucleation rate equation from the Classical Nucleation Theory shows that the pre-exponential kinetic term is orders of 10 magnitude lower than the theoretical value, possibly because either the attachment frequency or the concentration of heterogeneous particles is much lower than theoretically predicted. A thorough analysis of the deficit of Classical Nucleation Theory can be performed by using well-defined functionalized template particles in solutions having different kinds of self-association and solvation.

\section{${ }_{15}$ Acknowledgements}

The authors would like to acknowledge Shanfeng Jiang, Somnath Kadam, Nuria Llort Gual and Samir Kulkarni for doing the measurements in section 3 and discussing the data. They would further like to thank Gerard Coquerel, Samuel Petit, Roger Davey, Sven Schroeder and Hugo Meekes for interesting discussions on the

20 topic. JtH thanks the Dutch funding agency STW and the companies Avantium, DSM, Mettler-Toledo and Syncom for funding part of the research described here. $\mathrm{JtH}$ thanks the EPSRC Centre for Innovative Manufacturing in Continuous Manufacturing and Crystallisation (www.cmac.ac.uk) for supporting this work (EPSRC funding under grant reference: EP/I033459/1).

${ }^{a}$ Normandie Université, Crystal Genesis Unit, SMS, EA3233, IMR 4114 Université de Rouen, F76821 Mont Saint-Aignan Cedex, France. Current address: Particle Sciences, Devices \& Eng - UK $R \& D$ Platform Technology \& Science, GSK, Gunnels Wood Road, Stevenage, Hertfordshire, SG1 2NY,UK, Email: clement.m.brandel@gsk.com

$30{ }^{b}$ EPSRC Centre for Innovative Manufacturing in Continuous Manufacturing and Crystallisation (CMAC), Strathclyde Institute of Pharmacy and Biomedical Sciences, University of Strathclyde, 161 Cathedral Street, Glasgow G4 ORE, U.K. Tel: +44 141548 2858; Email:

Joop.terHorst@strath.ac.uk

\section{References}

1. S. Auer, D. Frenkel, Nature, 2001, 409, 1020-1023.

2. N.M. Dixit, A.M. Kulkarni, C.F. Zukoski, Coll. Surf. A: Physicochem. Eng. Aspects, 2001, 190, $47-60$

3. R.J. Davey, S.L.M. Schroeder, J.H. ter Horst, Nucleation of Organic Crystals - A Molecular Perspective, Angewandte Chemie, 2013, 52, 2166-2179.

4. O. Galkin, P.G. Vekilov, J. Phys. Chem. B, 1999, 103, 10965-10971.

5. P. G. Vekilov, Cryst. Growth Des. 2010, 10, 5007-5019.

6. S. Jiang, J.H. ter Horst, Crystal Nucleation Rates from Probability Distributions of Induction Times, Crystal Growth Design, 2011, 11, 256-261.

7. D. Kashchiev, Nucleation: basic theory with applications. Oxford: Butterworth-Heinemann; 2000.

8. S.S. Kadam, H.J.M. Kramer, J.H. ter Horst, Combination of a Single Primary Nucleation Event and Secondary Nucleation in Crystallization Processes, Crystal Growth Design, 2011, 11, $1271-1277$.

14 | [journal], [year], [vol], 00-00

This journal is @ The Royal Society of Chemistry [year] 
9. S.S. Kadam, S.A. Kulkarni, R. Coloma Ribera, A.I. Stankiewicz, J.H. ter Horst, Herman J.M. Kramer, A new view on the metastable zone width during cooling crystallization, Chem. Eng. Sci., 2012, 72, 10-19.

10. S.A. Kulkarni, H. Meekes, J.H. ter Horst, Polymorphism Control through a Single Nucleation Event, Crystal Growth Design, 2014, 14(3), 1493-1499.

11. S.A. Kulkarni, S.S. Kadam, H. Meekes, A.I. Stankiewicz, J.H. ter Horst, Crystal Nucleation Kinetics from Induction Times and Metastable Zone Widths, Crystal Growth Design, 2013, 13(6), 2435-2440.

12. U. J. Griesser, M.E. Auer, A. Burger, The Polymorphic Drug Substances of the European Pharmacopoeia, Part 10: Diprophylline, Sci. Pharm., 1999, 67, 319-330.

13. C. Brandel, Y. Amharar, J. Rollinger, U. Griesser, Y. Cartigny, S. Petit, G. Coquerel, Impact of Molecular Flexibility on Double Polymorphism, Solid Solutions and chiral Discrimination during Crystallization of Diprophylline Enantiomers, J. Molecular Pharmaceutics, 2013, 10(10), 3850-3861.

14. R. A. Sullivan, R. J. Davey, G. Sadiq, G. Dent, K. R. Back, J. H. ter Horst, D. Toroz, R. B. Hammond, Revealing the Roles of Desolvation and Molecular Self-Assembly in Crystal Nucleation from Solution: Benzoic and p-Aminobenzoic Acid, Crystal Growth Design, 2014, 14, 2689-2696.

15. D. Kashchiev, G.M. Van Rosmalen, Review: nucleation in solutions revisited, Cryst. Res. Technol., 2003, 38(7-8), 555.

16. A. Mersmann, J. Crystal Growth 1990, 102, 841

17. S.A. Kulkarni, E. S. McGarrity, H. Meekes, J.H. ter Horst, Isonicotinamide self-association: the link between solvent and polymorph nucleation, Chem Commun., 2012, 48, 4983-4985.

18. S.A. Kulkarni, C.C. Weber, A.S. Myerson, J.H. ter Horst, Self-association during heterogeneous nucleation onto well-defined templates, Langmuir, 2014, 30(41), 12368-12375. 